\title{
Characterization of OsPM19L1 encoding an AWPM-19-like family protein that is dramatically induced by osmotic stress in rice
}

\author{
H. Chen ${ }^{1}$, H. Lan ${ }^{2}$, P. Huang', Y. Zhang', X. Yuan'1, X. Huang ${ }^{1}$, J. Huang' ${ }^{1}$ and \\ H. Zhang ${ }^{1}$ \\ ${ }^{1}$ State Key Laboratory of Crop Genetics and Germplasm Enhancement/Jiangsu \\ Collaborative Innovation Center for Modern Crop Production, \\ Nanjing Agricultural University, Nanjing, China \\ ${ }^{2}$ College of Life Sciences, Nanjing Agricultural University, Nanjing, China \\ Corresponding authors: J. Huang / H. Zhang \\ E-mail: hszhang@njau.edu.cn / huangji@njau.edu.cn
}

Genet. Mol. Res. 14 (4): 11994-12005 (2015)

Received January 26, 2015

Accepted May 25, 2015

Published October 5, 2015

DOI http://dx.doi.org/10.4238/2015.October.5.12

ABSTRACT. The plant-specific AWPM-19-domain proteins play important roles in plant development and stress responses. In the current study, OsPM19L1 encoding Oryza sativa AWPM-19-like protein 1 was isolated from rice. Tissue-specific gene expression analysis revealed that OsPM19L1 was highly expressed in the leaf sheath of rice. Interestingly, expression of OSPM19L1 was high at the early stage of panicle development and decreased thereafter. qRT-PCR analysis indicated that OsPM19L1 was dramatically induced by $20 \%$ PEG stress (>600-fold), exogenous abscisic acid (>350-fold), salt and cold stress. Subcellular localization assay suggested that the OsPM19L1-GFP (green fluorescent protein) fusion protein was localized in the membrane system in rice cells. Moreover, under stress conditions, OsPM19L1 expression was enhanced in an $A B / 5$-Like1 ( $A B L 1)$ deficiency rice mutant, abl1, suggesting that ABL1 negatively regulates OsPM19L1 gene expression. Thus, OsPM19L1 
appears to be closely associated with stress tolerance through ABAdependent pathway in rice.

Key words: Rice; Membrane protein; AWPM-19-like; Abiotic stress; Panicle

\section{INTRODUCTION}

Drought, cold, and high salinity are major abiotic stresses that affect plant growth and crop production. In order to respond and adapt to these adverse factors, plants can initiate a number of molecular, cellular, and physiological changes. The stress-inducible genes play significant roles in response to these stresses (Kreps et al., 2002; Seki et al., 2002; Xiong et al., 2002; Zhu, 2002; Shinozaki et al., 2003; Lee et al., 2005). The phytohormone ABA plays a central role in the regulation of plant growth and developmental processes, as well as in the abiotic stress response, especially the response to drought or salt stress. ABA serves as an endogenous signal to initiate the adaptive responses when plants are challenged by abiotic or biotic stress (Zhu, 2002; Adie et al., 2007; Bari and Jones, 2009; Huang et al., 2012a; Ye et al., 2012; Nakashima and YamaguchiShinozaki, 2013; Danquah et al., 2014; Mehrotra et al., 2014). It has been established that two distinct pathways (ABA dependent and $A B A$ independent) are involved in stress-responsive gene expression (Huang et al., 2012b; Yoshida et al., 2014). Previous research has helped to identify the key components of ABA signaling. ABA signaling involves an ABA receptor complex, consisting of an $A B A$ receptor family, phosphatases and kinases. These proteins play a central role in regulating a variety of responses to diverse stresses, including the activities of membranelocalized factors (Osakabe et al., 2014). However, most studies at present focus on ABA functions in stress responses in Arabidopsis. Since there is limited insight into the specificity of rice signaling, studies on rice $A B A$ signal transduction will be very valuable to elucidate the functional mechanism of ABA. Rice ABI5-Like1 (ABL1), a basic region/leucine zipper motif transcription factor, modulates ABA responses by directly regulating the ABA-responsive element (ABRE)-containing genes (Zou et al., 2007, 2008; Yoshida et al., 2010; Yang et al., 2011).

Proteins with the AWPM-19 domain are classified into the AWPM-19-like family of proteins. The AWPM-19-like family proteins are widely distributed in various plant species. The roles for these proteins in seed development, dormancy and stress responses have been studied. GmPM3, the first identified AWPM-19-like family protein, was isolated from developing soybean seeds at the late embryogenesis stage, also classified as late embryogenesis-abundant (LEA) protein (Hsing et al., 1995). WPM-1, isolated by PCR from the library prepared from the ABA-treated cultured winter wheat (Triticum aestivum L. cv. Chihoku) cells, encodes a putative plasma membrane localized protein, WPM-1. Studies suggest that the WPM-1 promotes freezing tolerance of the wheat suspension-cultured cells by ABA treatment (Koike et al., 1997). The expression pattern of the PM19 gene, encoding a putative barley (Hordeum vulgare L.) plasma membrane protein, indicates that it is associated with embryo development, dormancy as well as with environmental stress during germination (Ranford et al., 2002). Besides soybean, wheat and barley, several AWPM-19 genes have been isolated from other plant species, such as Populus trichocarpa, Arabidopsis and rice, but the functional mechanisms for these proteins in plant development or stress tolerance are not clear.

In the present study, a novel AWPM-19-like family protein gene, OsPM19L1 was identified through microarray-based gene expression analysis in rice under ABA stress. Gene expression 
analysis suggested that OsPM19L1 was largely induced upon ABA stress and also induced by simulated drought, salt and cold stresses. Interestingly, OsPM19L1 expression was negatively regulated by an $\mathrm{ABI}$-like transcription factor, $\mathrm{ABL} 1$ under stress conditions, implying that OsPM19L1 might play a crucial role in osmotic stress response through the ABA-dependent pathway.

\section{MATERIAL AND METHODS}

\section{Plant materials and stress treatments}

The rice (Oryza sativa L. ssp japonica) cultivars Jiucaiqing, Zhonghua11 (ZH11), and abl1 mutant of Zhonghua11 (Yang et al., 2011) were used in this study. The seeds were sterilized in $0.1 \% \mathrm{HgCl}_{2}$ and germinated at $30^{\circ} \mathrm{C}$. The seedlings were cultured with Yoshida's solution in a growth chamber as previously described (Sun et al., 2012). All seedlings were kept under continuous light for $48 \mathrm{~h}$ before treatment and continuous light was used during the treatments. For stress and ABA treatments, the seedlings were cultured in Yoshida's solution supplemented with $100 \mathrm{mM} \mathrm{NaCl}, 20 \%$ PEG6000 (w/v), and $50 \mathrm{mM} \mathrm{ABA}$, respectively. For the cold treatment, seedlings were transferred to a growth chamber maintained at $4^{\circ} \mathrm{C}$. The seedling samples were collected at different time points after each treatment, immediately frozen in liquid nitrogen, and stored at $-80^{\circ} \mathrm{C}$. For tissue-specific gene expression analysis, the roots, stems, leaf blades, leaf sheathes, and panicles at different stages from adult plants were harvested and immediately frozen in liquid nitrogen.

\section{RNA isolation and first strand cDNA synthesis}

Total RNA was extracted from rice seedlings and different tissues treated with stress and ABA, using the Trizol reagent (Invitrogen, USA), as per manufacturer protocols. The RNA was subsequently treated with DNase I (Promega, USA) to remove the remaining genomic DNA. The first strand cDNA was synthesized with $2 \mu \mathrm{g}$ purified total RNA using the reverse transcription system (Promega).

\section{Cloning of OsPM19L1}

OSPM19L1 was cloned by PCR approach with a pair of primers (sense: 5'-GATTTGTGATTTTGATACGAGATGG-3' and antisense: 5'-TGCACGAGCGGGAAACAC-3'). The PCR conditions for amplifying OSPM19L1 were as follows: 5-min pre-denaturation at $98^{\circ} \mathrm{C}$, 28 cycles of $10 \mathrm{~s}$ at $98^{\circ} \mathrm{C}, 15 \mathrm{~s}$ at $56^{\circ} \mathrm{C}, 50 \mathrm{~s}$ at $72^{\circ} \mathrm{C}$, and a final extension for $10 \mathrm{~min}$ at $72^{\circ} \mathrm{C}$. The PCR product was purified and cloned into pEASY-T vector (TransGen, China) for sequencing.

\section{Quantitative real-time PCR (qRT-PCR)}

qRT-PCR was carried out on an Applied Biosystem 7500 Fast Real Time PCR System (Applied Biosystems). Quantification of gene expression was done using the comparative CT method. The 18S rRNA gene was used as an internal control (Jain et al., 2006). The primers of $18 S$ rRNA were: sense primer 5'-ATGGTGGTGACGGGTGAC-3' and antisense primer 5'-CAGACACTAAAGCGCCCGGTA-3'. 


\section{Multiple sequence alignment and phylogenetic analysis}

Alignment of OsPM19L1 and other plant AWPM-19-domain protein sequences were performed with the ClustalX program (ver 2.1). The phylogenetic tree was constructed with the MEGA program (ver 4.0) by neighbor-joining (NJ) method. The parameters pairwise deletion and $p$-distance model were used. Bootstrap test of phylogeny was performed with 1000 replicates.

\section{Subcellular localization}

A green fluorescent protein (GFP) fusion protein was constructed using the full-length OSPM19L1 cDNA clone, with an N-terminal fusion of the GFP clone under the control of CaMV 35 S promoter. Agrobacterium tumefaciens strain EHA105, containing an OSPM19L1::GFP fusion and a GFP alone was separately inoculated through the stomata using a 1-mL plastic syringe and by applying gentle pressure on the lower epidermal surface of tobacco leaves (Maselli et al., 2014). The subcellular distribution of the GFP fusion protein was examined by confocal laser scanning microscopy (Zeiss, LSM780).

\section{In silico sequence analysis}

For promoter sequence analysis, the 2000-bp upstream sequence of OSPM19L1 was analyzed by Matinspector program at Genomatix website (http://www.genomatix.de/). The coexpression analysis was performed both with Rice Oligo Array Database (http://ricearray.org/) based on 1081 rice Affymetrix microarray data (NCBI GEO AC: GPL2025) and by genevestigator (https://www.genevestigator.com/gv/). The genes coexpressed with OSPM19L1 were selected with a Pearson's correlation coefficient (PCC) cut-off of 0.6 in both results. The changes in expression of co-expressed genes under drought, salt, and cold stresses were investigated using GEO (Gene Expression Omnibus) datasets (http://www.ncbi.nlm.nih.gov) under accession No. GSE6901.

\section{RESULTS}

\section{Sequence analysis of OsPM19L1}

From microarray data on ABA-responsive genes in rice seedlings (Huang $\mathrm{J}$ and Zhang $\mathrm{H}$, unpublished data), an EST probe (probe ID: Os.11260.1.S1_at) showing 1220-fold induction upon exogenous ABA treatment was chosen for further analysis. Database search indicated that this EST encodes a putative AWPM-19-like family protein containing the AWPM-19 domain (Gene locus name: Os05g0381400). We designated this gene OsPM19L1 as it encodes an AWPM-19like family protein in rice. The full-length OsPM19L1 gene, containing a complete ORF of $522 \mathrm{bp}$, was cloned from total RNA prepared from rice seedlings by RT-PCR. The predicted protein product of OsPM19L1 comprised of 173 amino acids, with a calculated molecular mass of $18.06 \mathrm{kDa}$ and isoelectric point of 9.68. Multiple sequences alignment showed that OsPM19L1 was similar to other plant AWPM-19-domain-containing proteins (Koike et al., 1997; Ranford et al., 2002) (Figure 1). In particular, the N-terminal AWPM-19 domain is well conserved among OsPM19L1 and other AWPM-19-like proteins, whereas the C-terminal domain exhibits diversities. To investigate the evolutionary relationship among plant AWPM-19-like family proteins, a phylogenetic tree was 
constructed using NJ method with the full-length amino acid sequences of several AWPM-19-like family proteins - four members in rice (OsPM19L1, OsPM19L2, OsPM19L3, OsPM19L4), one in wheat (WPM-1), one in barley (pm19), one in sorghum (SbPM19L), one in Arabidopsis (AtPM19L), one in soybean (GmPM3), one in P. trichocarpa (PthPM19L), and one in peach (PpaPM19L). Phylogenetic analysis revealed that OsPM19L1 was clustered with WPM-1, PM19, and other AWPM-19-like proteins existing in rice and Sorghum bicolor, whereas others were categorized into another branch (Figure 2).

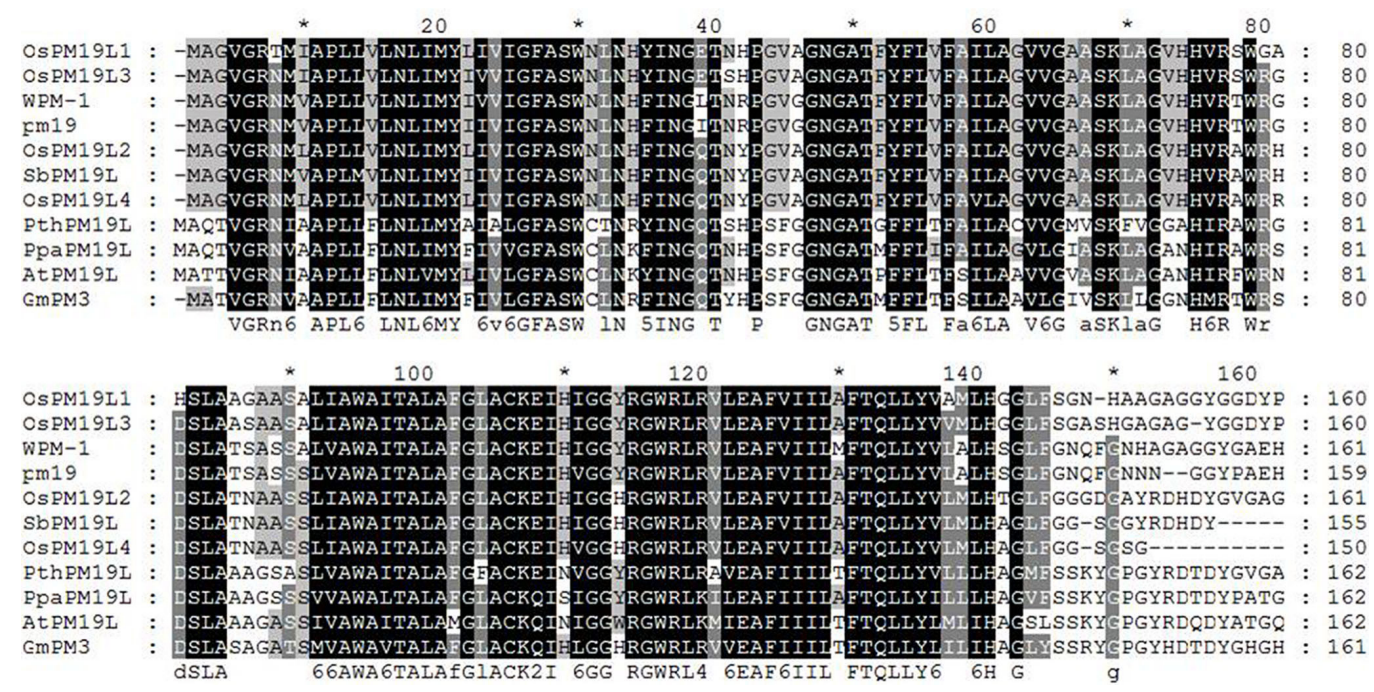

Figure 1. Multiple sequence alignment of AWPM-19-like family proteins. The amino acid sequences marked in black belong to conserved AWPM-19-domain. GenBank accession Nos. for the sequences: OsPM19L1 (NP_001055398.1), OsPM19L2 (NP_001059485.1), OsPM19L3 (XP_006654338.1), OsPM19L4 (XP_006658505.1), AWPM-19 (AAB38504.1), pm19 (AÄF29532.1), SbPM19L (XP_002459766.1), AtPM19L (NP_563710.1), GmPM3 (NP_001238105.1), PthPM19L (XP_002314514.1), PpaPM19L (XP_007223551.1).

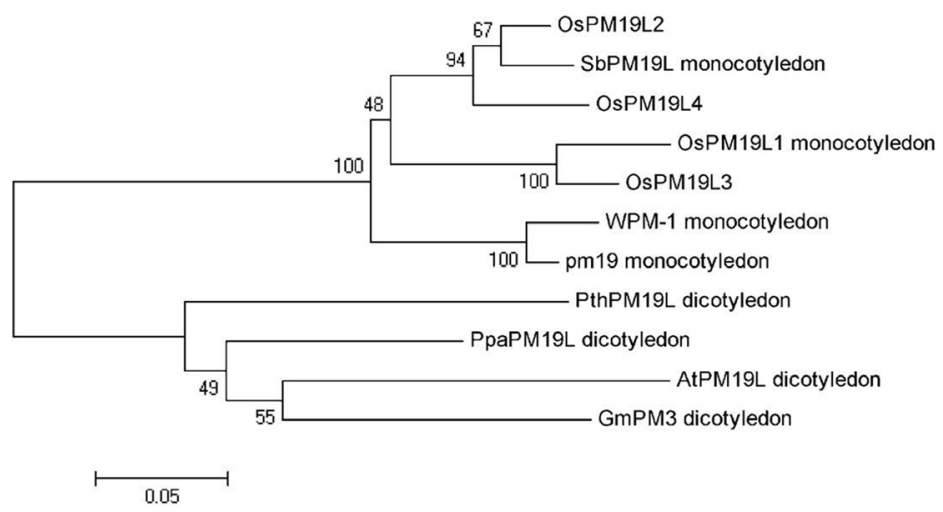

Figure 2. Phylogenetic analysis of AWPM-19-like family proteins. The neighbor-joining tree was constructed with MEGA 4.0. Branch numbers represent a percentage of the bootstrap values in 1000 sampling replicates and the scale bar indicates branch length. 
The 2000-bp promoter sequence of OsPM19L1 was cloned and analyzed through the MatInspector program (http://www.genomatix.de/). The promoter sequence contained some putative stress-related cis-acting elements, such as GTBX (GT-box elements) (Hamann et al., 1994), ABRE (Uno et al., 2000), DREB (dehydration responsive element binding factors) (Agarwal et al., 2006), MYBL (MYB-like proteins) (Abe et al., 2003), MYCL (MYC-likebinding factors), G-BOX (plant G-box/C-box bZIP proteins) (Figueroa and Browse, 2012), CGCG (calmodulin-binding/ CGCG box binding proteins) (Yang and Poovaiah, 2002), NACF (plant specific NAC transcription factors) (Takasaki et al., 2010), and WBXF (W Box family) (Figure 3). These stress-related cisacting elements may be responsive for stress-regulated expression of OsPM19L1.

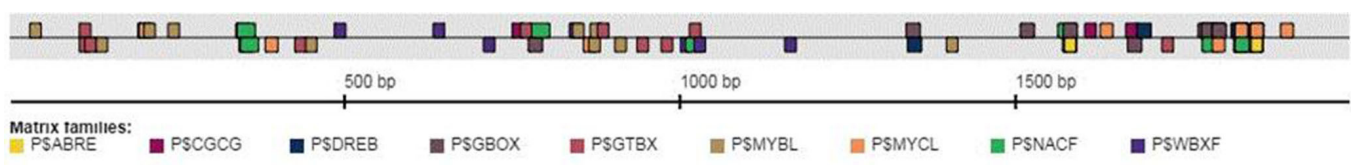

Figure 3. Distribution of stress-related cis-acting elements in the 2000 bp promoter region of OsPM19L1. Different cis-elements for TFs were labeled with different colors. Right end $2000 \mathrm{bp}$ is the transcriptional start site (TSS) of OsPM19L1.

\section{Expression analysis of OsPM19L1 in different rice tissues}

For tissue specific expression analysis, roots, stems, leaf blades, leaf sheathes, and panicles with lengths of $3 \mathrm{~cm}(\mathrm{P} 1), 8 \mathrm{~cm}(\mathrm{P} 2)$, and $12 \mathrm{~cm}(\mathrm{P} 3)$ were collected from adult rice plants. qRT-PCR results indicated that OsPM19L1 was constitutively expressed in various rice tissues, and predominately in leaf sheathes. We also investigated OsPM19L1 expression in panicles at different developmental stages, and found that the highest expression levels were in 3-cm young panicles, followed by a 4- and 5-fold decrease in expression levels in 8- and 12-cm panicles, respectively (Figure 4 ).

\section{Tissue Expression}

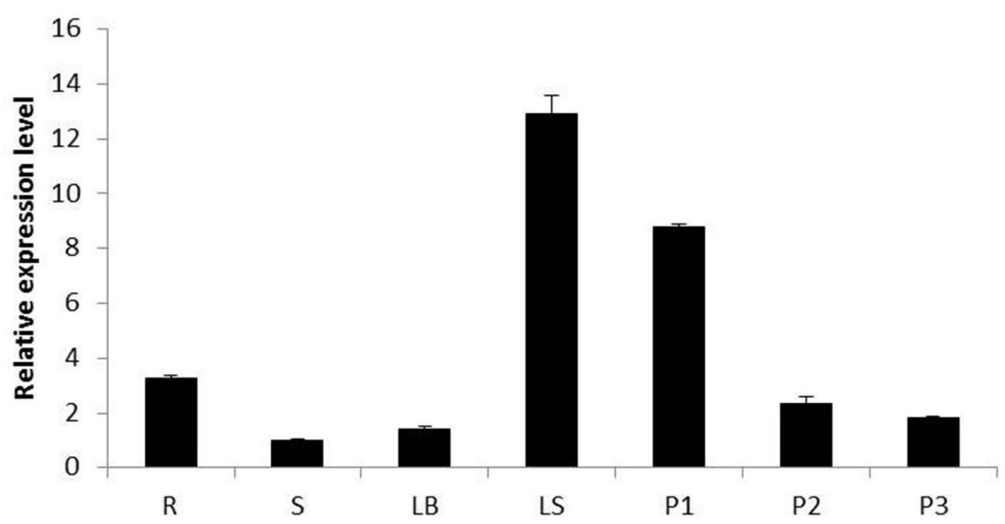

Figure 4. Expression pattern of OsPM19L1 in various tissues by real-time $\mathrm{qPCR}$. Roots (R), stems (S), leave blades (LB), leave sheathes (LS), and panicles with the length of $3 \mathrm{~cm}(\mathrm{P} 1), 8 \mathrm{~cm}(\mathrm{P} 2)$, and $12 \mathrm{~cm}(\mathrm{P} 3)$ in rice (Oryza sativa L. ssp japonica cultivar Jiucaiqing). 18S-rRNA was used as an internal control. Data are reported as means and standard errors of three replicates. 


\section{Induction of expression of OsPM19L1 in rice under abiotic stress}

Since the microarray data showed that OsPM19L1 was largely induced by ABA treatment, we investigated the expression pattern of the gene in response to various abiotic stresses. qRT-PCR assay showed that OsPM19L1 expression was highly induced by salt, PEG, low temperature, and ABA treatment. For the $100-\mathrm{mM} \mathrm{NaCl}$ treatment, OsPM19L1 transcript started responding to the treatment after $3 \mathrm{~h}$, and showed two-peak induction pattern at 6- and 24-h treatment with a decrease in expression at $12 \mathrm{~h}$ (Figure 5D). The transcript levels of OSPM19L1 increased remarkably after $3 \mathrm{~h}$ of $20 \%$ PEG treatment, and compared to control, a 660 -fold increase at $12 \mathrm{~h}$ was observed (Figure 5B). After 50- $\mu \mathrm{M}$ exogenous ABA treatment, OSPM19L1 expression in seedlings showed a 360-fold increase at $12 \mathrm{~h}$, which remained at a similar level till $24 \mathrm{~h}$, while a 95-fold increase was obsreved at $48 \mathrm{~h}$ (Figure 5A). For the low temperature treatment, OsPM19L1 expression changes were not as dramatic as those observed for other stresses, and the OsPM19L1 transcript accumulated slowly, starting to respond to the stress after $24 \mathrm{~h}$ (Figure $5 \mathrm{C}$ ).

A

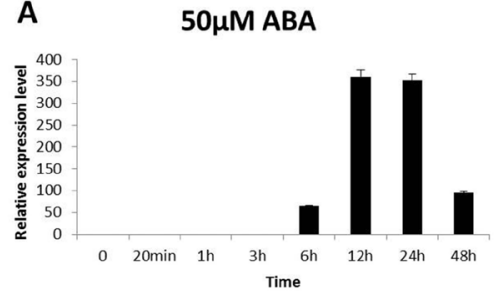

C

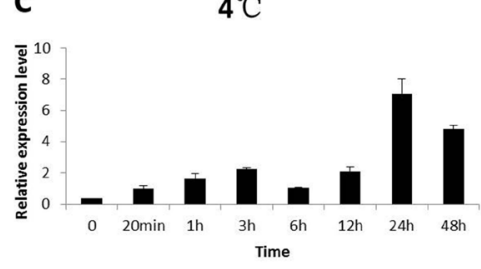

B
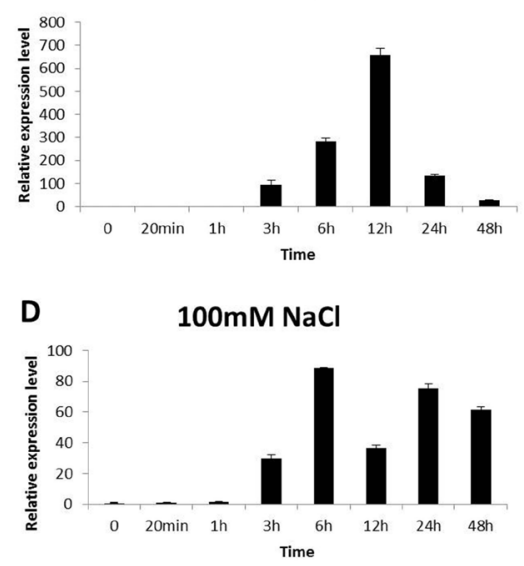

Figure 5. Expression patterns of OsPM19L1 in rice seedlings treated with various stresses and ABA by real-time qPCR. A. $50 \mu \mathrm{M}$ ABA, B. cold stress $\left(4^{\circ} \mathrm{C}\right)$, C. $100 \mathrm{mM} \mathrm{NaCl}$, D. stimulated drought stress (20\% PEG). Three to four leaf rice seedlings (Jiucaiqing) were used. 18S-rRNA was used as an internal control. Data are reported as means and standard errors of three replicates.

\section{Expression of OsPM19L1 in rice abl1 mutant under PEG and $\mathrm{NaCl}$ stress}

The rice ABI5-like1 (ABL1) deficiency mutant, ab/1, shows suppressed ABA responses (Yang et al., 2011). Since previous data suggest that OsPM19L1 expression is regulated by ABA treatment, it was of interest to analyze whether OsPM19L1 was involved in ABL1 signaling pathway. OsPM19L1 gene expression patterns in ab/1 and wild-type rice $\mathrm{ZH} 11$ under $\mathrm{NaCl}$ and PEG stresses were examined. Compared to untreated samples, a 2140-fold increase in OsPM19L1 expression was observed in ab/1 after $12 \mathrm{~h}$ of $20 \%$ PEG6000 treatment, while in WT a 276-fold increase was observed. A similar trend was also observed for $\mathrm{NaCl}$ treatment, where after $12 \mathrm{~h}$ of treatment, OsPM19L1 expression in ab/1 was 25-fold higher than in untreated samples. OsPM19L1 expression was up-regulated greatly in ab/1 mutant under PEG and $\mathrm{NaCl}$ treatment (Figure 6A-C). 
A

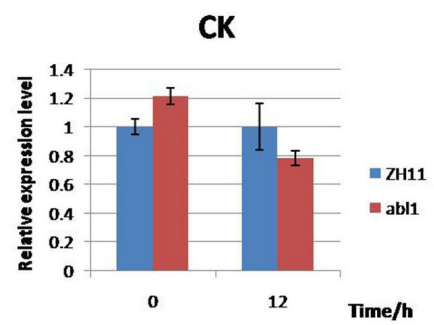

B

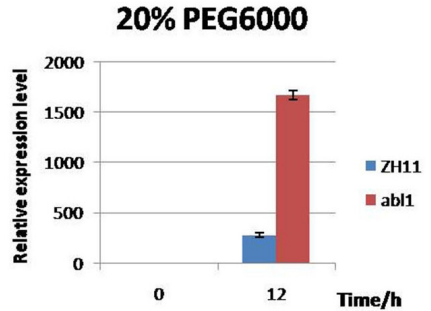

C

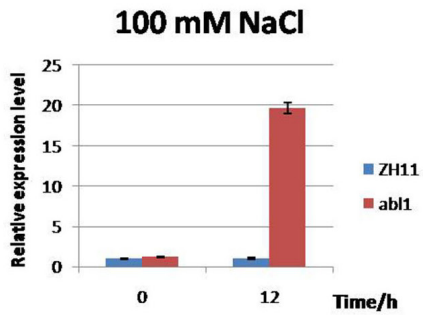

Figure 6. Expression change of OsPM19L1 in rice ab/1 mutant and WT (ZH11) seedlings under different treatments. A.-C. Expression of OsPM19L1 in the seedlings exposed to water (CK), stimulated drought stress (20\% PEG6000) and $100 \mathrm{mM} \mathrm{NaCl}$, respectively. 18S-rRNA was used as an internal control. Data are reported as means and standard errors of three replicates.

\section{Co-expression analysis of OsPM19L1}

The identities of seven genes, that were selected from the OSPM19L1 co-expression gene data as having PCC higher than 0.6, provide clues about the functions of OsPM19L1 in plant stress responses (Table 1). These genes might function coordinately in response to abiotic stress. Six of the seven genes (RAB16D, RAB16B, RAB21, Os05g0349800, Os06g0246500, and Os03g0322900) showed highly up-regulated response to all three abiotic stresses. Interestingly, only Os03g0111300, a lipid-transfer protein, was induced by drought and salt treatments and inhibited by cold stress.

Table 1. List of genes co-expressed with OsPM19L1.
\begin{tabular}{llccrr}
\hline Gene ID & Annotation & PCC & Drought & Salt & Cold \\
\hline Os11g0453900 & Dehydrin Rab16D & 0.7765 & $5.81 \pm 0.43$ & $5.18 \pm 0.77$ & $0.07 \pm 1.21$ \\
Os11g0454200 & Dehydrin Rab16B & 0.7304 & $9.54 \pm 0.20$ & $7.34 \pm 0.33$ & $0.06 \pm 0.76$ \\
Os11g0454300 & Water stress-inducible protein Rab21 & 0.6882 & $8.04 \pm 0.35$ & $7.19 \pm 0.35$ & $0.20 \pm 0.36$ \\
Os05g0349800 & Late embryogenesis abundant protein & 0.6736 & $6.68 \pm 0.20$ & $3.35 \pm 0.60$ & $1.83 \pm 1.88$ \\
Os03g0111300 & Nonspecific lipid-transfer protein 2 (nsLTP2) & 0.6467 & $3.35 \pm 0.36$ & $3.59 \pm 0.52$ & $-2.04 \pm 2.66$ \\
Os06g0246500 & Pyruvate dehydrogenase E1 alpha subunit & 0.643 & $4.23 \pm 0.34$ & $3.60 \pm 0.77$ & $0.85 \pm 1.13$ \\
Os03g0322900 & Late embryogenesis abundant protein & 0.636 & $7.41 \pm 0.10$ & $6.33 \pm 0.41$ & $2.52 \pm 1.99$ \\
\hline
\end{tabular}

PCC = Pearson's correlation coefficient. The data in the table indicate the log2 fold of the microarray data in rice seedlings under cold, drought, or salt stress relative to that under control condition. The normalized microarray data were generated from GEO database under accession No. GSE6901. Standard error was based on three biological repeats.

\section{OsPM19L1 locates in the membrane system}

As OsPM19L1 encodes a putative AWPM-19-domain protein, it was of interest to investigate whether OsPM19L1 was a membrane protein. We analyzed the subcellular localization of OsPM19L1 using the transient expression system in tobacco leaves. OsPM19L1GFP fusion protein was localized in the organelle membranes, while the control GFP protein was detected throughout the cell, indicating that the GFP protein remained in the cytosol (Figure 7). 

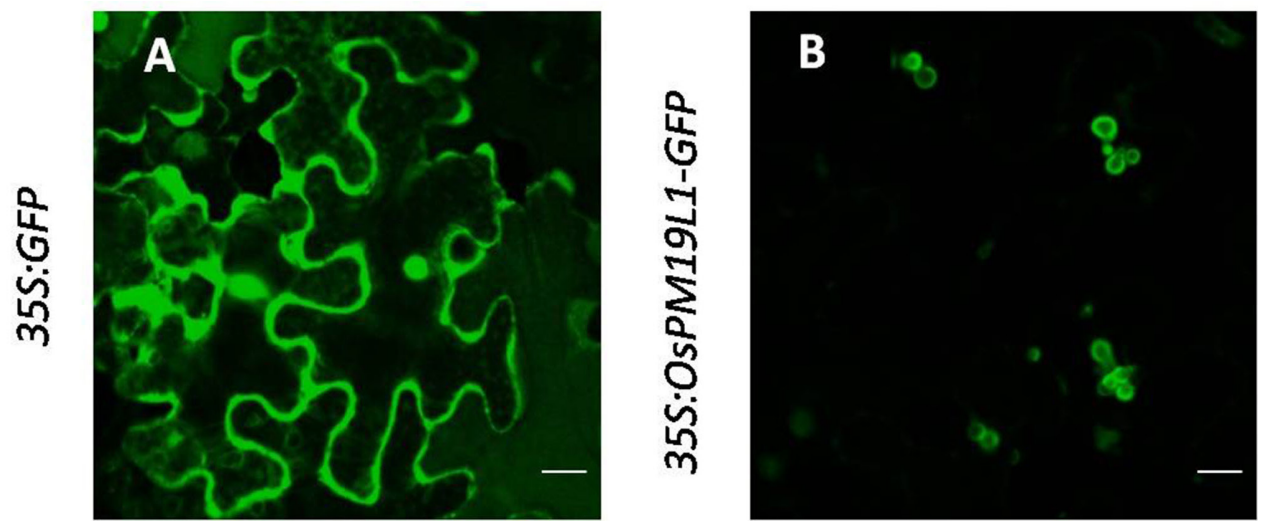

Figure 7. Subcellular localization of OsPM19L1 in tobacco epidermal cells. Each Agrobacterium tumefaciens strain EHA105 containing the 35S:GFP (control empty vector) (A), 35S:OsPM19L1-GFP (B) proteins was transiently expressed in Nicotiana benthamiana leaves. (bar $=20 \mu \mathrm{m})$.

\section{DISCUSSION}

In present study, we report a novel AWPM-19-like family protein gene OsPM19L1, whose expression was dramatically induced by simulated drought, osmotic stress and exogenous ABA. Through multiple sequence alignment ang phylogenetic analysis, it was found that OsPM19L1 is mostly related to wheat protein WPM-1 and barley protein pm19. These two plants both belong to monocotyledons. The phylogenetic analysis indicated that the AWPM-19 gene family might be formed by the species-specific gene expansion. The results of tissue specific expression analysis indicate that OsMP19L1 is highly expressed at the early stage of panicle development and probably plays an essential role in immature panicles. However, this hypothesis needs further validation using OsMP19L1 knock-out mutant. MYB transcription factors are important for normal reproductive development of plants. Overexpression of cotton GhMYB24 in Arabidopsis led to flower malformation, shorter filaments, non-dehiscent anthers and fewer viable pollen grains ( $\mathrm{Li}$ et al., 2013), while, in transgenic rice, supra-optimal expression of OsMyb4 transcription factor caused mis-expression of alternative targets with costly trade-offs to panicle development (Park et al., 2010). Since there is a MYB-like protein-binding site in the promoter region of OsMP19L1, it will be of interest to investigate the functions of OsMP19L1 in plant reproductive development.

Co-expression analysis shows a number of genes involved in abiotic stress coexpressed with OsPM19L1. The top three genes (RAB16D, RAB16B, RAB21) and Os06g0246500, encoding pyruvate dehydrogenase E1 alpha subunit, are well-documented stress-induced ABA late responsive genes (Wang et al., 2011). Two genes (Os05g0349800 and Os03g0322900) encoding LEA proteins are highly induced by drought, salt and cold stresses. Many LEA proteins are known to be involved in abiotic stress tolerance in plant (Dalal et al., 2009). Collectively, these coexpression results suggest that OSPM19L1 plays a positive role in drought salinity and cold tolerance by regulating stress-induced $A B A$ response in rice.

Gene expression analysis indicated that OsPM19L1 was significantly induced by salt and PEG stresses but not markedly by cold, implying that OsPM19L1 is mainly involed in signal transduction induced by osmotic stress. Based on the observation that OsPM19L1 was dramatically induced by $A B A$ treatment, it may play an important role in ABA signaling. The $A B L 1$ 
protein is localized to the nucleus and modulates the plant stress and ABA responses by directly regulating the ABRE-containing genes (Yang et al., 2011). An ABRE protein binding site in the promoter region of OsPM19L1 suggests that OsPM19L1 may participate in plant stress and ABA responses under the regulation of $A B L 1$ transcription factor. Although the role of OsPM19L1 in ABA signal transduction is not yet understood, it provides an interesting avenue for further study of rice OsPM19L1 functions in stress response via the ABA dependent pathway.

The subcellular localization results suggest that OSPM19L1 is a membrane protein. The features of WPM-1 amino acid composition indicate that WPM-1 is a membrane protein (Koike et al., 1997). Membrane-delimited events play a crucial role in ABA signaling, and ABA receptors (PYR/PYL/RCAR) modulate the activity of different plasma membrane components involved in ABA action (Bueso et al., 2014).

In conclusion, a new AWPM-19-like family protein gene OsPM19L1 was identified and characterized in present study. OsPM19L1 was localized in plasma membrane and endomembrane system, implying that OsPM19L1 functions as a membrane-localized protein in ABA signaling pathway. Interestingly, we found that expression of OsPM19L1 was regulated by ABL1. As OsPM19L1 expression was largely induced upon drought, osmotic stress and exogenous ABA application, we hypothesize that OsPM19L1 is involved in stress response via an ABA-dependent pathway in rice. However, this hypothesis needs further experimental validation by use of OsPM19L1 knock-out mutant or transgenic approach.

\section{Conflicts of interests}

The authors declare no conflicts of interests.

\section{ACKNOWLEDGMENTS}

Research supported by the Natural Science Foundation of China (\#31071069), Natural Science Foundation of Jiangsu Provience (\#BK20141362), the "111" Project, and the Fundamental Research Funds for the Central Universities (\#KYZ201137).

\section{REFERENCES}

Abe H, Urao T, Ito T, Seki M, et al. (2003). Arabidopsis AtMYC2 (bHLH) and AtMYB2 (MYB) function as transcriptional activators in abscisic acid signaling. Plant Cell 15: 63-78.

Adie BA, Perez-Perez J, Perez-Perez MM, Godoy M, et al. (2007). ABA is an essential signal for plant resistance to pathogens affecting JA biosynthesis and the activation of defenses in Arabidopsis. Plant Cell 19: 1665-1681.

Agarwal PK, Agarwal P, Reddy MK and Sopory SK (2006). Role of DREB transcription factors in abiotic and biotic stress tolerance in plants. Plant Cell Rep. 25: 1263-1274.

Bari R and Jones JD (2009) Role of plant hormones in plant defence responses. Plant Mol. Biol. 69: 473-488.

Bueso E, Rodriguez L, Lorenzo-Orts L, Gonzalez-Guzman M, et al. (2014). The single subunit RING-type E3 ubiquitin ligase RSL1 targets PYL4 and PYR1 ABA receptors in plasma membrane to modulate abscisic acid signaling. Plant J. 80: 1057-1071.

Dalal M, Tayal D, Chinnusamy V and Bansal KC (2009). Abiotic stress and ABA-inducible Group 4 LEA from Brassica napus plays a key role in salt and drought tolerance. J. Biotechnol. 139: 137-145.

Danquah A, de Zelicourt A, Colcombet J and Hirt H (2014). The role of ABA and MAPK signaling pathways in plant abiotic stress responses. Biotechnol. Adv. 32: 40-52.

Figueroa P and Browse J (2012). The Arabidopsis JAZ2 promoter contains a G-Box and thymidine-rich module that are necessary and sufficient for jasmonate-dependent activation by MYC transcription factors and repression by JAZ proteins. Plant Cell Physiol. 53: 330-343. 
Hamann L, Bayer KU, Jensen K and Harbers K (1994). Interaction of several related GC-box- and GT-box-binding proteins with the intronic enhancer is required for differential expression of the gb110 gene in embryonal carcinoma cells. Mol. Cell Biol. 14: 5786-5793.

Hsing YIC, Chen ZY and Chow TY (1995). A soybean cDNA (accesssion No. L20806) encoding a hydrophobic embryogenesis abundant protein. Plant Physiol. 109: 1125-1127.

Huang GT, Ma SL, Bai LP, Zhang L, et al. (2012a). Signal transduction during cold, salt, and drought stresses in plants. Mol. Biol. Rep. 39: 969-987.

Huang P, Ju HW, Min JH, Zhang X, et al. (2012b). Molecular and physiological characterization of the Arabidopsis thaliana oxidation-related zinc finger 2, a plasma membrane protein involved in ABA and salt stress response through the ABI2mediated signaling pathway. Plant Cell Physiol. 53: 193-203.

Jain M, Nijhawan A, Tyagi AK and Khurana JP (2006). Validation of housekeeping genes as internal control for studying gene expression in rice by quantitative real-time PCR. Biochem. Biophys. Res. Commun. 345: 646-651.

Koike M, Takezawa D, Arakawa K and Yoshida S (1997). Accumulation of 19-kDa plasma membrane polypeptide during induction of freezing tolerance in wheat suspension-cultured cells by abscisic acid. Plant Cell Physiol. 38: 707-716.

Kreps JA, Wu Y, Chang HS, Zhu T, et al. (2002). Transcriptome changes for Arabidopsis in response to salt, osmotic, and cold stress. Plant Physiol. 130: 2129-2141.

Lee BH, Henderson DA and Zhu JK (2005). The Arabidopsis cold-responsive transcriptome and its regulation by ICE1. Plant Cell 17: 3155-3175.

Li Y, Jiang J, Du ML, Li L, et al. (2013). A cotton gene encoding MYB-like transcription factor is specifically expressed in pollen and is involved in regulation of late anther/pollen development. Plant Cell Physiol. 54: 893-906.

Maselli GA, Slamovits CH, Bianchi Jl, Vilarrasa-Blasi J, et al. (2014). Revisiting the evolutionary history and roles of protein phosphatases with Kelch-like domains in plants. Plant Physiol. 164: 1527-1541.

Mehrotra R, Bhalothia P, Bansal P, Basantani MK, et al. (2014). Abscisic acid and abiotic stress tolerance - different tiers of regulation. J. Plant Physiol. 171: 486-496.

Nakashima K and Yamaguchi-Shinozaki K (2013). ABA signaling in stress-response and seed development. Plant Cell Rep. 32: 959-970.

Osakabe Y, Yamaguchi-Shinozaki K, Shinozaki K and Tran LS (2014). ABA control of plant macroelement membrane transport systems in response to water deficit and high salinity. New Phytol. 202: 35-49.

Park MR, Yun KY, Mohanty B, Herath V, et al. (2010). Supra-optimal expression of the cold-regulated OsMyb4 transcription factor in transgenic rice changes the complexity of transcriptional network with major effects on stress tolerance and panicle development. Plant Cell Environ. 33: 2209-2230.

Ranford JC, Bryce JH and Morris PC (2002). PM19, a barley (Hordeum vulgare L.) gene encoding a putative plasma membrane protein, is expressed during embryo development and dormancy. J. Exp. Bot. 53: 147-148.

Seki M, Narusaka M, Ishida J, Nanjo T, et al. (2002). Monitoring the expression profiles of 7000 Arabidopsis genes under drought, cold and high-salinity stresses using a full-length cDNA microarray. Plant J. 31: 279-292.

Shinozaki K, Yamaguchi-Shinozaki K and Seki M (2003). Regulatory network of gene expression in the drought and cold stress responses. Curr. Opin. Plant Biol. 6: 410-417.

Sun $\mathrm{H}$, Huang X, Xu X, Lan H, et al. (2012). ENAC1, a NAC transcription factor, is an early and transient response regulator induced by abiotic stress in rice (Oryza sativa L.). Mol. Biotechnol. 52: 101-110.

Takasaki H, Maruyama K, Kidokoro S, Ito Y, et al. (2010). The abiotic stress-responsive NAC-type transcription factor OsNAC5 regulates stress-inducible genes and stress tolerance in rice. Mol. Genet. Genomics 284: 173-183.

Uno Y, Furihata T, Abe H, Yoshida R, et al. (2000). Arabidopsis basic leucine zipper transcription factors involved in an abscisic acid-dependent signal transduction pathway under drought and high-salinity conditions. Proc. Natl. Acad. Sci. U. S. A. 97: 11632-11637.

Wang D, Pan Y, Zhao X, Zhu L, et al. (2011). Genome-wide temporal-spatial gene expression profiling of drought responsiveness in rice. BMC Genomics 12: 149.

Xiong L, Schumaker KS and Zhu JK (2002). Cell signaling during cold, drought, and salt stress. Plant Cell 14 (Suppl): S165-S183.

Yang T and Poovaiah BW (2002). A calmodulin-binding/CGCG box DNA-binding protein family involved in multiple signaling pathways in plants. J. Biol. Chem. 277: 45049-45058.

Yang X, Yang YN, Xue LJ, Zou MJ, et al. (2011). Rice ABI5-Like1 regulates abscisic acid and auxin responses by affecting the expression of ABRE-containing genes. Plant Physiol. 156: 1397-1409.

Ye N, Jia L and Zhang J (2012). ABA signal in rice under stress conditions. Rice 5: 1.

Yoshida T, Fujita Y, Sayama H, Kidokoro S, et al. (2010). AREB1, AREB2, and ABF3 are master transcription factors that cooperatively regulate $A B R E$-dependent $A B A$ signaling involved in drought stress tolerance and require $A B A$ for full activation. Plant J. 61: 672-685. 
Yoshida T, Mogami J and Yamaguchi-Shinozaki K (2014). ABA-dependent and ABA-independent signaling in response to osmotic stress in plants. Curr. Opin. Plant Biol. 21: 133-139.

Zhu JK (2002). Salt and drought stress signal transduction in plants. Annu. Rev. Plant Biol. 53: 247-273.

Zou M, Guan Y, Ren H, Zhang F, et al. (2007). Characterization of alternative splicing products of bZIP transcription factors OsABI5. Biochem. Biophys. Res. Commun. 360: 307-313.

Zou M, Guan Y, Ren H, Zhang F, et al. (2008). A bZIP transcription factor, OsABI5, is involved in rice fertility and stress tolerance. Plant Mol. Biol. 66: 675-683. 